\title{
Scaling Exponents and Multifractal Dimensions for Independent Random Cascades
}

\author{
G.M. Molchan \\ International Institute of Earthquake Prediction and Mathematical Geophysics, Moscow, 113556, \\ Russia
}

Received: 12 September 1995/Accepted: 23 January 1996

\begin{abstract}
This paper is concerned with Mandelbrot's stochastic cascade measures. The problems of (i) scaling exponents of structure functions of the measure, $\tau(q)$, and (ii) multifractal dimensions are considered for cascades with a generator vector $\left(w_{1} \cdots w_{c}\right)$ of the general type. These problems were previously studied for independent strongly bounded variables $w_{i}: 0<a<w_{i} \leqq c$. Consequently, a broad class of models used in applications, including Kolmogorov's log-normal model in turbulence, log-stable "universal" cascades in atmospheric dynamics, has not been covered. Roughly speaking, problems (i), (ii) are here solved under the condition that the scaling exists; the $\tau$-function is calculated for all arguments (previously this was done for positive $q$ ) and a new effect emerges: the $\tau$-function can generally involve discontinuities in the first derivative as well as in the second.
\end{abstract}

\section{Introduction and Results}

Independent random cascades and cascade measures first arose as simple models of fully developed turbulence [10]. They describe the space distribution of energy dissipation rate in the transmission of energy from coarser to finer vortices. In recent years random cascades are being intensively used for theoretical interpretations of empirical data in various natural sciences [14,15]. A gap has formed by now between theoretical models that have been investigated $[1,8]$ and what is of practical interest. We are going to study the multifractal characteristics of cascade measures under natural conditions of existence for these measures. This is in the first place Renyi's $\tau$-function or scaling exponents of structure functions of different orders for a cascade measure. The function occupies a central place in the statistical theory of turbulence. The solution presented here is not complete, but the results enable one to determine $\tau(q),|q|<\infty$, provided the scaling exists, and to detect a generally possible loss of continuity in the derivative $\tau(q)$ (this has not been known before). We extend Kahane's result [9] about the existence of moments of the total mass of a cascade measure to the case of negative exponents.

We begin with definitions. 
Let

$$
W=\left(w_{1} \cdots w_{c}\right), \quad w_{i} \geqq 0
$$

be a random vector where $c$ is a cascade branching number. Consider an infinite sequence of independent copies of $W$ on the probability space $(\Omega, \mathscr{A}, \mathscr{P})$ in order to construct independent random functions $P_{n}(t)$ on $J=[0,1]$. Define $P_{n}$ recursively:

$$
P_{1}(t)=\sum_{1 \leqq i \leqq c} w_{i} \chi_{\Delta_{i 1}},
$$

where $\left\{\Delta_{i 1}\right\}$ is a partition of $J$ into " $c$ " equal intervals and $\chi_{\Delta}$ is the indicator function of $\Delta$; then,

$$
P_{n+1}((i+\tau) / c)=P_{n}^{(i)}(\tau), \quad 0 \leqq \tau<1, i=0,1, \ldots, c-1,
$$

where $P_{n}^{(i)}$ are independent copies of $P_{n}$. (In order to emphasize that $P_{n}$ depends on the distribution of $W$, we shall also use the slightly incorrect notation $P_{n}(t \mid W)$ or $P_{n}(t \mid w)$, if components of $W$ are i.i.d.) Consider

$$
\mu_{n}(d t)=\prod_{i=1}^{n} P_{i}(t) d t
$$

as a stochastic measure on $J$. The total mass of $\mu_{n}, M_{n}$, is obviously equal to 1 on the average, if

$$
E \frac{1}{c} \sum w_{i}=1
$$

If the sequence of $\mu_{n}$ weakly converges a.s. to a non-trivial measure $\mu$, i.e., $\mu(d t) \neq d t$ and $E \mu(J)=1$, then we will deal with an independent random cascade with random cascade measure $(\mu \mid W)$. We distinguish three types of cascades: general type (2), Mandelbrot's cascade (briefly $\mathscr{M}$-cascade) which has i.i.d. components of $W$ with mean 1 , and conservative cascades having the property:

$$
\sum_{1 \leqq i \leqq c} w_{i} / c=1 \quad \text { a.s } .
$$

As is well known [9], an $\mathscr{M}$-cascade has a non-trivial measure $\mu$ with $P(M>0)=1, M=\mu(J)$, iff

$$
E w \log _{c} w<1, \quad P(w>0)=1, \quad P(w=1)<1, \quad E w=1 .
$$

The support of the $\mathscr{M}$-cascade measure is a fractal set of Hausdorff dimension $d=1-E w \log _{c} w$, iff $E w(\log w)^{2}<\infty[9,17]$.

A cascade measure is a multifractal. The study of its multifractal properties reduces to:

(i) determination of the Rényi function

$$
\tau(q)=\underset{n \rightarrow \infty}{\operatorname{Lim} \log } \sum_{i} \mu^{q}\left(\Delta_{i n}\right) / \log \Delta_{n}, \quad \Delta_{n}=\left|\Delta_{\text {in }}\right|,
$$

where the limit needs to be specified and $\left\{\Delta_{i n}\right\}, i=1, \ldots, c^{n}$ is partition of $J$ into $c^{n}$ equal intervals;

(ii) a substantiation of the multifractal formalism, namely, the Legendre transform of the $\tau$-function

$$
\mathscr{L} \tau(\alpha)=\min _{q}(q \alpha-\tau(q)):=f(\alpha)
$$


should have the meaning of the fractal dimension of the set

$$
J_{\alpha}=\left\{t \in J: \lim _{n \rightarrow \infty} \log \mu\left(\Delta_{n}(t)\right) / \log \Delta_{n}=\alpha\right\}
$$

where $\Delta_{n}(t)$ is the sequence of intervals $\Delta_{i n}$ that contain $t$. We shall also use the notation $J_{\alpha}(\mathscr{N})$ if the limit in (5) is taken on some subsequence $\mathscr{N}$ of $Z_{+}=$ $\{1,2, \ldots\}$.

1.1. $\mathscr{M}$-cascade: Renyi function $\tau(q)$. The study of $M$-cascades is central to the problem in hand. An approximation to the $\tau$-function of an $\mathscr{M}$-cascade can be obtained by substituting successively in (4):

$$
\mu\left(\Delta_{\text {in }}\right) \rightarrow \mu_{n}\left(\Delta_{\text {in }}\right) \rightarrow E \mu_{n}\left(\Delta_{\text {in }}\right) .
$$

The first substitution is justified by appealing to the finite range of scales experimentally observed, while the second is equivalent to the passage from spatial averaging to that over all samples of $\mu$.

Then (4) reduces to the function $\hat{\tau}(q)=-\Phi(q)$, where

$$
\Phi(q)=\log _{c} E w^{q}-q+1 .
$$

In the statistical theory of turbulence, the above approximation is usually employed for theoretical scaling exponents (4), provided $\Phi(q)$ is defined for all $q>0$ and $\dot{\Phi}(q)<0$ ("Novikov's inequalities" [12]).

The question of when and where $\tau(q)=\hat{\tau}(q), q>0$ and what is the correct version of $\hat{\tau}$ has been investigated in recent papers $[1,8]$ for a very restricted class of models:

$$
0<a<w<c \text { a.s }
$$

The case (7) does not cover the classical example of turbulent cascades related to the log-normal hypothesis of A. Kolmogorov. In this example $\log w$ is gaussian, therefore $w$ is not bounded either from 0 or from $\infty$ (to say nothing of the bound c), even though it has "light tails," i.e., $E w^{q}<\infty$ for any $|q|<\infty$.

Recently the statistics of multifractal processes tends to make use of the socalled "universal" class of cascades in which $\log w$ is an infinitely divisible (in particular, Levy-stable) random variable $[12,14,16]$. In this case $\hat{\tau}(q)$ can be finite only on some part of $R^{1}$, so that the question as to the relation between $\tau$ and $\hat{\tau}$ becomes especially urgent for interpretation of experimental data.

Before formulating the result for the $\tau$-function, let us consider the graph of the convex function $\Phi(q)$ (including infinite values). Under (3) it is the boundary of the convex set $G=\{(q, y): y \geqq \Phi(q)\}$, the points $(0,1)$ and $(1,0)$ belong to $G$ but $(0,0) \notin G$. Consequently, the smallest closed cone containing $G$ has the boundaries $\Gamma_{+}=\left\{\left(q, c_{+} q\right), q>0\right\}$, and $\Gamma_{-}=\left\{\left(q, c_{-} q\right), q<0\right\}$, where $c_{ \pm} \leqq 0$. By (3), $\Phi(q)(|\Phi|<\infty)$ is analytic and different from a linear function. Hence the intersection $\Gamma_{ \pm}$with $\Phi(q)$ either consists of a single point with the abscissa $q_{ \pm}$or is empty (with the understanding that $q_{ \pm}= \pm \infty$ ). Obviously, if $\left|q_{ \pm}\right|<\infty$, then $\Gamma_{ \pm}$ is the support line for $G$ and $q_{+} \geqq 1, q_{-} \leqq 0$.

The points $q_{ \pm}$where the support lines and $\Phi(q)(\Phi<\infty)$ are transversal will here be called points of the first kind, while all the other points will be ones of the second kind. 
Define $\Phi_{\star}(q)$ by

$$
\Phi_{\star}(q)= \begin{cases}\Phi(q), & q_{-}<q<q_{+} \\ c_{ \pm} q, & q / q_{ \pm}>1, \quad q_{-} \neq 0 . \\ \infty & q_{-}=0\end{cases}
$$

Theorem 1. Under the conditions (3) there exists a sequence of integer numbers $\mathscr{N}=\left\{n_{i}\right\}$ such that

$$
\lim _{\mathscr{N} \ni n \rightarrow \infty} \frac{1}{n} \log \sum_{1 \leqq i \leqq c^{n}} \mu^{q}\left(\Delta_{\text {in }}\right)=\Phi_{\star}(q), \quad \text { a.s . }
$$

for all q, i.e., the limit (9) holds for samples of the measure $\mu(\cdot \mid \omega), \omega \in \Omega^{\prime}$, $\mathscr{P}\left(\Omega^{\prime}\right)=1$, where $\Omega^{\prime}$ does not depend on $q$.

Remark. If the scaling (4) exists in probability then $\tau(q)=-\Phi_{\star}(q)$. In addition, it follows from the proof of Theorem 1 that if the scaling relation (4) exists a.s. for all $q \in\left(q_{-}, q_{+}\right)$and $q_{+}\left(q_{-}\right)$is a point of the second kind, then $\tau(q)=-\Phi_{\star}(q)$ for all $q>q_{-}\left(q<q_{+}\right)$.

In [1], the existence of the limit and the form of $\tau(q)$ for $q>0$ were established under the conditions (7). In that case $\Phi(q)$ is bounded for all $q$ and $\Phi_{\star}(q)$ extends $\Phi(q), q \in\left(0, q_{+}\right)$beyond the critical point $q_{+}<\infty$ of the tangent line at $q=$ $q_{+}-0$. In the general case (3) the support line $y=c_{+} q$ for the $\Phi(q)$-graph ceases to be tangent, if $q_{+}$is a boundary of the finite values of $\Phi$. For this reason the $\tau$-function (if it exists) can have discontinuities in the first derivative.

1.2. $\mathscr{M}$-cascades: multifractal dimensions. The critical points $q_{ \pm}$of two types generate two types of $\alpha$-intervals:

with

$$
\left(\alpha_{-}^{(2)}, \alpha_{+}^{(2)}\right) \subseteq\left(\alpha_{-}^{(1)}, \alpha_{+}^{(1)}\right)
$$

$$
\alpha_{\mp}^{(2)}=-\dot{\Phi}\left(q_{ \pm} \mp 0\right), \quad \alpha_{\mp}^{(1)}=\Phi\left(q_{ \pm} \mp 0\right) /\left(q_{ \pm}-0\right) .
$$

If $\left|q_{ \pm}\right|=\infty$, then the right-hand sides of (11) are to be understood as the limits with $q \rightarrow q_{ \pm}= \pm \infty$.

In the intervals (10) the Legendre transform of the $\tau$-function, $f(\alpha)$, is defined and is strictly and non-strictly convex, respectively ( $f$ is linear in the complement of the first interval).

Theorem 2. (a) Lower bound. Under (3) for any fixed $\alpha \in\left(\alpha_{-}^{(2)}, \alpha_{+}^{(2)}\right), f(\alpha) \neq 1$, the following estimate holds:

$$
H-\operatorname{dim} J_{\alpha} \geqq f(\alpha)
$$

if $q_{-} \neq 0$. Let $q_{-}=0$ and $E|\log w|^{\varepsilon}<\infty$ for some $\varepsilon>0$, then (12) is true for $J_{\alpha}(\mathscr{N})$, where $\mathscr{N}$ is any sequence of type $\left\{n_{i}: n_{i+1} / n_{i}>\rho>1\right\}$;

(b) Upper bound. Let $\mathscr{N}=\left\{n_{i}\right\}$ be the same as in Theorem 1. Then under the condition (3)

$$
H-\operatorname{dim} J_{\alpha}(\mathscr{N}) \leqq f(\alpha), \quad \alpha \in\left(\alpha_{-}^{(1)}, \alpha_{+}^{(1)}\right) \quad \text { a.s. },
$$

i.e., (13) holds for $\mu(\cdot \mid \omega), \omega \in \Omega^{\prime}, P\left(\Omega^{\prime}\right)=1$ and $\Omega^{\prime}$ does not depend on $\alpha$. 
From Theorems 1,2 it follows that, if (4) exists a.s. for $q \in\left(q_{-} q_{+}\right)$, where $q_{ \pm}$ are critical points of the second kind and $q_{-}<0$, then

$$
H-\operatorname{dim} J_{\alpha}=f(\alpha), \quad \alpha: f(\alpha) \neq 0,1, \quad \text { a.s . }
$$

All the above conditions are met under (7), [1]. Consequently, (14) holds for strongly bounded cascades (7). Certain particular results on these lines were derived in $[8,13]$.

The second part of Theorem $2 \mathrm{a}$ permits one to analyze multifractal dimensions of $\mathscr{M}$-cascades having a Levy-stable distribution for $\log w$ with the parameters $\alpha_{L} \in(0,2)$ and $\beta_{L}=1$. In that case $E|\log w|^{\varepsilon}<\infty \forall \varepsilon \in\left(0, \alpha_{L}\right)$ and

$$
\Phi(q)=\left\{\begin{array}{ll}
\infty, & q<0 \\
(1-D)\left(q^{\alpha_{L}}-q\right) /\left(\alpha_{L}-1\right)-q+1, & q>0
\end{array},\right.
$$

where $D \in(0,1)$ is the dimension of the cascade measure carrier. Among the critical points

$$
q_{-}=0, \quad q_{+}=(1-D)^{-1 / \alpha_{L}}
$$

there is a point $q_{\star}$ of the first kind, iff $\alpha_{L} \in(1,2)$ and $q_{\star}=q_{-}$. If the scaling (4) exists a.s. for all $q \in\left(0, q_{+}\right)$then Theorems 1,2 lead to the relation (14) with $J_{\alpha}(\mathcal{N})$, where $\mathcal{N}=\left\{n_{i}: n_{i+1} / n_{i}>\rho>1\right\}$. In fact, if $\alpha_{L}>1$, then $f(\alpha)=1$ for all $\alpha>\alpha_{+}^{(2)}=\left(\alpha_{L}-D\right) /\left(\alpha_{L}-1\right)$. Hence the requirement $f \neq 1$ excludes the interval $\left(\alpha_{+}^{(2)}, \alpha_{+}^{(1)}\right)$, while the interval $\left(\alpha_{-}^{(1)}, \alpha_{-}^{(2)}\right)$ is empty.

1.3. Cascades of the general type. Let $W=\left(w_{1}, \ldots, w_{c}\right)$ be a cascade vector with the components $w_{i} \geqq 0, E \sum w_{i} / c=1$. Consider the random component of $W$, namely

$$
w^{(r)}=\left\{w_{i} \text { with probability } 1 / c, \quad i=1, \ldots, c .\right.
$$

The function $\Phi(q)$ corresponding to $w^{(r)},\left(\Phi^{(r)}(q)\right.$, say ) plays the same part for a cascade of the general type as (6) does for an $\mathscr{M}$-cascade.

Theorem 3. Theorems 1, 2 with $w=w^{(r)}$ and $\Phi=\Phi^{(r)}$ remain valid for the cascade measure $(\mu \mid W)$.

In other words, the multifractal characteristics $(\tau, f)$ do not distinguish between cascades of $\mathscr{M}$ type and conservative type.

1.4. Auxiliary propositions. The proofs of Theorems 1 through 3 are based on well-known results for the solution of the following stochastic equation which the total mass $M$ of measure $\mu$ obeys:

$$
M=\frac{1}{c} \sum_{1 \leqq i \leqq c} w_{i} M_{i}
$$

where the $M_{i}$ are independent copies of $M$ that are independent of $W=\left\{w_{i}\right\}$ as well $[3,6,7]$. Also needed are results for the moments of $M$. The requirements for existence of the positive moments of $M$ were derived in $[9,11]$, while some information on the negative moments is furnished by the following. 
Theorem 4. Let $w$ be the random component of a cascade vector $W$. Then under (3), the following implications are true:

(a) $E w^{-h}<\infty \Rightarrow E M^{-h a}<\infty, \quad h>0$,

(b) $E|\log w|^{h}<\infty \Rightarrow E|\log M|^{h^{\prime} a}<\infty, \quad \forall h^{\prime} \in(0, h)$,

where $a=c$ for $\mathscr{M}$-cascades and $a=1$ in the general case (2).

The constant " $a$ " in Theorem $4(a)$ is exact. We give two examples. Let the random component of $W$ have the beta-distribution with parameters $(h, h(c-1))$, that is,

$$
P\left(w^{(r)}<x\right)=k \cdot x^{h}(1+o(1)), \quad h \rightarrow 0 .
$$

Then $M$ for an $\mathscr{M}$-cascade has the gamma-distribution with the parameter $c \cdot h$, i.e.

$$
P(M<x)=k_{1} x^{c h}(1+o(1)), \quad x \rightarrow 0,
$$

[6]. Consequently, $E w^{q}<\infty, q>-h$, while $E M^{q}<\infty$ for $q>-c h$.

The value $M$ for the cascade with $W=(w, \ldots, w)$ will also have the gammadistribution, but the parameter is $h$ [7]. Hence, $E M^{-h^{\prime}}<\infty, \forall h^{\prime}<h$.

Theorems 1,2,3 are proved in Sects. 2,3,4, respectively. Theorem 4 is proved in Sects. 2 and 4.

Below we use the following notation:

$\mathscr{P}$-lim, $\stackrel{\mathscr{P}}{\rightarrow}$ means convergence in probability;

$d$-lim, $\stackrel{d}{\rightarrow}$ means convergence in distribution;

$H$-dim is Hausdorff dimension.

\section{2. $\tau$-Function for $\mathscr{M}$-Cascades: Proof of Theorems 1,4}

The proof is given as a sequence of independent propositions. Of these, Proposition 4 contains proof of Theorem 4 for $\mathscr{M}$-cascades.

Proposition 1. If $M$ is the total mass of the cascade measure $(\mu \mid w, c)$, then

$$
M=c^{-1} \sum_{1 \leqq i \leqq c} w_{i} M_{i}
$$

where $\left(w_{i}, M_{i}\right)$ are independent copies of $(w, M)$ and $w, M$ are independent as well. Any nontrivial nonnegative solution of (15) has the property $P(M>0)=1$, if $P(w>0)=1$.

Proof. Equation (15) is well known [10] and follows from the definition of $M$. By (15),

$$
P(M=0)=[P(w M=0)]^{c},
$$

whence $P(M=0)=0$, if $P(w>0)=1$.

Proposition $2[7,6]$. Let $E w^{q}<\infty$ for some $q>1$ and $\xi \geqq 0$ be a random variable with $E \xi=1$. Then under (3) one has

$$
M_{n}(\xi):=M_{n}(w, \xi):=\int P_{1}(t \mid w) \cdots P_{n}(t \mid w) P_{n}(t \mid \xi) d t \stackrel{d}{\rightarrow} M
$$


as $n \rightarrow \infty$. Here, $P_{n}(t \mid \xi)$ is constructed in the same way as is $P_{n}(t \mid w)$ and is independent of $P_{i}(t \mid w), i \leqq n$.

Proposition 3 [9]. Under (3) one has $E M^{q}<\infty \forall q \in[0,1]$; for $q>1$,

$$
E M^{q}<\infty \Leftrightarrow E w^{q}<\infty \text { and } \Phi(q)<0 \text {. }
$$

Proposition 4. For $q<0$ under (3), the following implications are true:

(a) $E w^{q}<\infty \Rightarrow E M^{q c}<\infty$,

(b) $E|\log w|^{|q|}<\infty \Rightarrow E|\log M|^{h c}<\infty, \quad \forall h<|q|$.

Proof of Proposition 4a. Let $E M^{-\varepsilon}<\infty$ for some $\varepsilon>0$. By (15) one has

$$
M^{-1}=c / \sum_{1 \leqq i \leqq c} w_{i} M_{i} \leqq \prod_{l}\left(M_{i} w_{i}\right)^{-1 / c} .
$$

Hence

$$
E M^{-q}<\left(E M^{-q / c}\right)^{c}\left(E w^{-q / c}\right)^{c}, \quad q>0 .
$$

Putting $q / c=\varepsilon$, one gets $E M^{-c \varepsilon}<\infty$, if $E w^{-q_{0}}<\infty, q_{0} \geqq \varepsilon$. Repeating the above argument with $q<c^{n} \varepsilon, n=2,3, \ldots$, one obtains $E M^{-q_{0} c}<\infty$.

Consequently, it suffices to show that $E M^{-\varepsilon}<\infty$, if $E w^{-q_{0}}<\infty$. Let

$$
\varphi(s)=E \exp (-s M) \text {. }
$$

By (15),

$$
\varphi(s)=\left[\int_{0}^{\infty} \varphi(s x) d F(c x)\right]^{c},
$$

where $F$ is the distribution of $w$. Divide the $x$-axis into two parts by the point $x_{0}=s^{-1 / 2}$ and use the following estimates:

$$
\varphi(s x) \leqq 1, \quad x<x_{0} ; \quad \varphi(s x) \leqq \varphi\left(s^{1 / 2}\right), \quad x>x_{0} .
$$

Then by (17) we have

$$
\varphi(s)<\left[F\left(c s^{-1 / 2}\right)+\varphi\left(s^{1 / 2}\right)\right]^{c} .
$$

Using Chebyshev's inequality,

$$
F(x)=P\left(w^{-1}>x^{-1}\right) \leqq E\left(w^{-q_{0}}\right) \cdot x^{q_{0}} .
$$

Therefore

$$
\varphi\left(s^{2}\right)<\left[m s^{-q_{0}}+\varphi(s)\right]^{c} .
$$

Since $P(M>0)=1$ (Proposition 1), $\varphi(s)=o(1), s \rightarrow \infty$. For this reason, for $c>2$ and $s$ that is large enough,

$$
\varphi\left(s^{2}\right)<p\left[s^{-h}+\varphi(s)\right]^{2}, \quad s>s_{0}, \quad h<q_{0}, \quad p<1,
$$

where

$$
p=\max _{s \geqq s_{0}}\left[m s^{-q_{0}}+\varphi(s)\right]^{c-2}=o(1), \quad s_{0} \rightarrow \infty .
$$

Let us make (19) somewhat cruder:

$$
\varphi\left(s^{2}\right)<2 p\left(s^{-2 q}+\varphi^{2}(s)\right) \quad s>s_{0} .
$$


Putting $\psi(u)=\varphi(\exp (u))$, one has

$$
\psi(2 u)<2 p\left(e^{2}(u)+\psi^{2}(u)\right), \quad e(u):=\exp (-q u),
$$

where $u=\ln s$. Iterating (20), we shall make use of the estimate

$$
(a+b)^{2}<2\left(a^{2}+b^{2}\right) .
$$

One then gets

$$
\psi\left(2^{n} u\right)<a_{n}[e(u)]^{2^{n}}+b_{n} \cdot[\psi(u)]^{2^{n}},
$$

where the coefficients satisfy the recurrence relations

$$
\begin{gathered}
b_{n+1}=4 p b_{n}^{2}, \quad b_{1}=2 p, \\
a_{n+1}=2 p+4 p a_{n}^{2}, \quad a_{1}=2 p .
\end{gathered}
$$

Hence

$$
4 p b_{n+1}=\left(4 p b_{n}\right)^{2}
$$

and

$$
b_{n}=(2 \sqrt{2} p)^{2^{n}} / 4 p=2^{-1 / 2}
$$

if $p=2^{-3 / 2}$.

If $\hat{a}_{n}=\sqrt{2} a_{n}$, then

$$
\hat{a}_{n+1}=1+\hat{a}_{n}^{2}, \quad \hat{a}_{1}=1 .
$$

It is easy to see that

$$
\hat{a}_{n}<Q^{2^{n}}, \quad 1<Q<2 .
$$

Hence

$$
\psi\left(x_{n} u\right)<\exp \left(-x_{n}\left(u-u_{\star}\right)\right)+[\psi(u)]^{x_{n}}, \quad x_{n}:=2^{n},
$$

where $u_{\star}=\ln Q$. Let $u>\max \left(u_{\star}, \ln s_{0}\right)$ and

$$
x_{n} u<x<x_{n+1} u .
$$

Then

$$
\begin{gathered}
\psi(x)<\psi\left(x_{n} u\right), \\
\exp \left(-x_{n}\left(u-u_{\star}\right)\right)<\exp \left(-x\left(1-u_{\star} / u\right) / 2\right), \\
\psi(u)^{x_{n}}<\psi(u)^{x /(2 u)} .
\end{gathered}
$$

Consequently,

$$
\psi(x)<\exp (-x \alpha)+\psi(u)^{x /(2 u)}, \quad \alpha=\left(1-u_{\star} / u\right) / 2
$$

or

$$
\varphi(s)<s^{-\alpha}+s^{-\beta}, \quad s>s_{0},
$$

where

$$
\beta=|\ln \psi(u)| /(2 u) .
$$

It remains to be observed that

$$
\int_{0}^{\infty} s^{h-1} \varphi(s) d s=\Gamma(h) E M^{-h}, \quad h>0 .
$$


By virtue of (21) the left-hand side of (22) is finite for small $h$. Therefore, the same is true for the right-hand side as well.

We have assumed that $c>2$. This restriction can be removed by iterating (18).

Proof of Proposition $4 b$. Similarly to the above,

$$
\varphi\left(s^{2}\right)<[F(c / s)+\varphi(s)]^{c}<2^{c-1}\left[F^{c}\left(c s^{-1}\right)+\varphi^{c}(s)\right] .
$$

If $E|\log w|^{\alpha}<\infty$, then $F(x)<k|\log x|^{-\alpha}, x<1$.

Let $1<c^{\prime}<c$. Choosing a suitable $s_{0}$, one gets

$$
\varphi\left(s^{2}\right)<p\left[\left(\ln \frac{1}{s}\right)^{-\alpha c^{\prime}}+\varphi^{c^{\prime}}(s)\right], \quad s>s_{0},
$$

where

$$
p=p\left(s_{0}, c^{\prime}\right)=o(1), \quad s_{0} \rightarrow \infty .
$$

Putting $\psi(u)=\varphi\left(e^{u}\right)$, one has

$$
\psi(2 u)<p\left(u^{-\alpha c^{\prime}}+\psi^{c^{\prime}}(u)\right), \quad u>u_{0} .
$$

We will show by induction that

$$
\psi\left(2^{n}\right) \leqq\left(2^{n-n_{0}}\right)^{-\alpha c^{\prime}}, \quad n \geqq n_{0}, \quad 2^{n_{0}}>u_{0} .
$$

When $n=n_{0}$, the inequality is obvious. Pass from $n$ to $n+1$ using (23):

$$
\psi\left(2^{n+1}\right) \leqq p\left[\left(2^{n}\right)^{-\alpha c^{\prime}}+\left(2^{n-n_{0}}\right)^{-\alpha c^{\prime 2}}\right]=k\left(2^{n-n_{0}+1}\right)^{-\alpha c^{\prime}}
$$

where

$$
k=p\left[2^{-\left(n_{0}-1\right) \alpha c^{\prime}}+\left(2^{n-n_{0}}\right)^{-\alpha\left(c^{\prime 2}-c^{\prime}\right)} 2^{\alpha c^{\prime}}\right]<p\left(1+2^{\alpha c^{\prime}}\right) .
$$

If $p<\left(1+2^{\alpha c^{\prime}}\right)^{-1},(24)$ is proven. Similarly to the above, this yields the estimate

$$
\varphi(s)<k(\log s)^{-\alpha c^{\prime}}, \quad \forall c^{\prime}<c, s>s_{0},
$$

where $k$ depends on $c^{\prime}$ and $s_{0}$.

By the Tauber theorems [5],

$$
\rho \int_{1}^{\infty} e^{-s x} s^{-1}|\ln s|^{\rho-1} d s=|\ln x|^{\rho}(1+o(1)), \quad x \rightarrow 0, \rho>0 .
$$

Integrating both parts of this relation with respect to $d F(x)$ ( $F$ is the distribution of $M$ ), one gets

$$
\int^{\infty} \varphi(s) s^{-1}|\ln s|^{\rho-1} d s<\infty \Rightarrow \int_{0}|\ln x|^{\rho} d F(x)<\infty, \quad \rho=\alpha c^{\prime \prime},
$$

where $1<c^{\prime \prime}<c^{\prime}<c$. Therefore

$$
E|\log w|^{\alpha}<\infty \Rightarrow E|\log M|^{\alpha c^{\prime \prime}}<\infty, \quad \forall c^{\prime \prime} \in(1, c) .
$$

\section{Proposition 5. Under (3)}

(a) the cascade variables

$$
w_{q}=w^{q} / E w^{q}
$$


generate cascade measures with total mass $M_{q}: E M_{q}=1, P\left(M_{q}>0\right)=1$, iff $q \in\left(q_{-}, q_{+}\right) \cup \Gamma$, where $\Gamma$ consists of points $q_{ \pm}$of the first kind.

If $q \in\left(q_{-}, q_{+}\right)$, then $E M_{q}^{h}<\infty$ with $h=h(q)>1$.

(b) $E M^{q}<\infty$ if $q \in\left(q_{-}, q_{+}\right) \cup\left\{q_{ \pm}:\left|q_{ \pm}\right|<\infty\right\}$.

Proof. By [9], $E M_{q}=1$, iff

$$
f:=E w_{q} \log _{c} w_{q}-1<0 .
$$

But

$$
f(q)=q \dot{\Phi}(q)-\Phi(q),
$$

whence $\dot{f}(q)=q \ddot{\Phi}(q)$, where $\ddot{\Phi}(q) \geqq 0$ because the moment function is logconvex. Therefore $f(q)$ increase with increasing $|q|, f(0)=-1$. For this reason (25) is valid in some interval $\Delta=\left(k_{-}, k_{+}\right) \ni 0$.

We now show that $k_{ \pm}=q_{ \pm}$. The function $f(q)(\Phi<\infty)$ has a simple geometrical interpretation:

$-f(q)$ is the point of intersection of the line

$$
y=\Phi(q)+\dot{\Phi}(q)(x-q)
$$

tangent to the convex curve $\Phi(\cdot)$ at $q \in\left(Q_{-}, Q_{+}\right)$with the $x=0$ axis. Here $\left(Q_{-}, Q_{+}\right)$is the domain of finite values for $\Phi(q)$. Let $Q_{-}<0$, otherwise $Q_{-}=0$ and $q_{-}=k_{-}=0$. Since $-f(q)$ decreases with $|q| \rightarrow \infty$, three cases can arise.

Case 1. As $|q|$ increases, $-f(q)$ reaches 0 . Then the tangent becomes identical with the support line passing through the origin. Therefore $k_{\delta}=q_{\delta}(\delta=+$ or -$), q_{\delta}$ is a point of the second kind and $w_{q}$ does not satisfy (25) at $q=q_{\delta}$.

Case 2. When $q$ varies in the intervals $\left(0, Q_{+}\right)$or $\left(Q_{-}, 0\right)$, the function $-f(q)$ reaches the limiting level of $f_{0}>0$. Since $f(q)$ is analytic in $\left(Q_{-}, Q_{+}\right)$and is nonlinear because $w \neq$ const, we conclude that $k_{\delta}=Q_{\delta}$. But then, the line connecting $(0,0)$ with $\left(Q_{\delta}, f\left(Q_{\delta}\right)\right)$ supports $\Phi(q)$, i.e. $k_{\delta}=Q_{\delta}=q_{\delta}$ and $q_{\delta}$ is a point of the first kind.

Since $f\left(q_{\delta}\right)<0, E M_{q}=1$ (Proposition 3).

Case 3. $-f(q)$ reaches 0 or $f_{0}>0$ asymptotically, so then $k_{\delta}=Q_{\delta}$. We show that $\left|Q_{\delta}\right|=\infty$. Suppose the opposite is true. Then $0<\left|Q_{\delta}\right|<\infty$ and $f(q)=q \dot{\Phi}(q)-$ $\Phi(q)$ has a finite one-sided limit as $q \rightarrow Q_{\delta}$. Since $\Phi$ is analytic, this is possible, if $\Phi(q)$ and $\dot{\Phi}(q)$ simultaneously tend to finite or infinite limits. The first possibility is not considered, because it is relevant to cases 1 and 2 . It follows that $|\Phi(q)| \rightarrow \infty$, $q \rightarrow Q_{\delta}$

Since $\Phi$ is convex, $\Phi(q) \rightarrow \infty, \delta \dot{\Phi}(q) \rightarrow \infty$ with $q \rightarrow q_{\delta}$. Let $\delta=+1$. Then there exists a point $q_{0}>1$, where $\Phi(q)$ reaches the minimum and therefore $\Phi(q)>\phi\left(q_{0}\right)=-f\left(q_{0}\right)>0=\Phi(1), q>0$. This is a contradiction. Let $\delta=-1$. Since $q=Q$ is the vertical asymptote of $\Phi$ and $\Phi(q), q \in\left(Q_{-}, 0\right)$ is convex, we can find the line $y=\beta q$ tangent to $\Phi$ at a point $q_{0} \in\left(Q_{-}, 0\right)$, i.e. $f\left(q_{0}\right)=0$. This is a contradiction again.

So, $\left|Q_{\delta}\right|=\infty$ and $\dot{\Phi}(q), \delta q>0$ has the asymptote $y=f_{0}+\beta_{\delta} q, f_{0} \geqq 0$. Therefore $y=\beta_{\delta} q, \delta q>0$ is a part of the boundary of the cone containing $\Phi$. Hence $\left|q_{\delta}\right|=\infty$, i.e. $q_{\delta}=k_{\delta}$. 
We now show that $\exists h>1: E M_{q}^{h}<\infty$, if $q \in\left(q_{-}, q_{+}\right)$. The functions $\Phi$ for the cascade variables $w$ and $w_{q}$, namely, $\Phi$ and $\Phi_{q}$, are connected by

$$
\Phi_{q}(h)=\Phi(h q)-h \Phi(q) \text {. }
$$

Hence

$$
\begin{aligned}
\Phi_{q}(h) & =-(h-1)(\Phi(q)-\dot{\Phi}(q) q)+o(h-1) \\
& =f(q)(h-1)+o(h-1) .
\end{aligned}
$$

Since $f(q)<0, q \in\left(q_{-}, q_{+}\right), \Phi_{q}(h)<0$ for $1<h<h_{0}(q)$ and Proposition 3 gives $E M_{q}^{h}<\infty$.

Proposition $5 \mathrm{~b}$ is a corollary of Propositions 3 and 4.

Proposition 6. (a) If $q \in\left(q_{-}, q_{+}\right)$, then under (3),

$$
d-\lim _{n \rightarrow \infty} \sum_{1 \leqq i \leqq c^{n}} \mu^{q}\left(\Delta_{i n}\right) c^{-n \Phi(q)}=M_{q} E M^{q},
$$

where $M_{q}$ is the total mass of the cascade measure for the variable $W_{q}$, $P\left(M_{q}>0\right)=1$;

(b) There exists a sequence of integer numbers $\mathscr{N}=\left\{n_{i}\right\}$ such that the pointwise convergence

$$
S_{n}(q):=\frac{1}{n} \log _{c} \sum_{i} \mu^{q}\left(\Delta_{i n}\right) \rightarrow \Phi(q), \quad \forall q \in\left(q_{-}, q_{+}\right)
$$

takes place as $n \rightarrow \infty, n \in \mathscr{N}$ for samples of the measure $\mu(d t \mid \omega), \omega \in \Omega^{\prime}$ with $\mathscr{P}\left(\Omega^{\prime}\right)=1$.

Proof. By the definition of $\mu$,

$$
\mu\left(\Delta_{n}(t)\right)=P_{1}(t) \cdots P_{n}(t) P_{n}(t \mid M) \Delta_{n},
$$

where $\Delta_{n}(t)=\Delta_{i n} \ni t, P_{n}(t \mid M)$ is a function of type (1) that is independent of $P_{i}(t), i \leqq n$. Hence

$$
\sum_{n}(q):=\sum_{i} \mu^{q}\left(\Delta_{i n}\right)=\int P_{1}^{q}(t) \cdots P_{n}^{q}(t) P_{n}^{q}(t \mid M) \Delta_{n}^{q-1} d t .
$$

Using the normalization

$$
P_{i}^{q}(t) / E w^{q}=P_{i}\left(t \mid w_{q}\right), \quad M^{q} / E M^{q}=\xi,
$$

one gets

$$
\sum_{n}(q)=\int P_{1}\left(t \mid w_{q}\right) \cdots P_{n}\left(t \mid w_{q}\right) P_{n}(t \mid \xi) d t \cdot c_{n} \cdot E M^{q}
$$

where

In the notation (16),

$$
c_{n}=c^{n \Phi(q)} .
$$

$$
c_{n}^{-1} \sum_{n}(q)=M_{n}\left(w_{q}, \xi\right) \cdot E M^{q} .
$$

Let $q \in\left(q_{-}, q_{+}\right)$, then (see Proposition 5) $E M_{q}^{h}<\infty, 1<h=h(q)$; the cascade variables $w_{q}$ generate cascade measures with $M_{q}^{q}: E M_{q}=1, P\left(M_{q}>0\right)=1 ; E M^{q}<\infty$ and $M \xi=1$. The use of Proposition 2 gives (26). By (26) and $P\left(M_{q}>0\right)=1$,

$$
L_{n}(q)=\frac{1}{n} \log _{c}\left(\sum_{n}(q) \cdot c_{n}^{-1}\right) \stackrel{\mathscr{P}}{\rightarrow} 0, \quad n \rightarrow \infty .
$$


Let $\mathscr{R}$ be a denumerable, everywhere dense subset of $\left(q_{-}, q_{+}\right)$. Then one can choose a subsequence $\left\{n_{i}\right\}=\mathscr{N}$ and a set $\Omega^{\prime}$ of events $\{\omega\}$ such that $P\left(\Omega^{\prime}\right)=1$ and

$$
S_{n}(q)=\Phi(q)+L_{n}(q) \rightarrow \Phi(q), \quad q \in \mathscr{R}, \omega \in \Omega^{\prime},
$$

where $n \in \mathscr{N}$ and $n \rightarrow \infty$. We are going to show that (29) holds on the completion of $\mathscr{R}$.

The upper bound of $S_{n}(q \mid \omega), n \in \mathcal{N}$ for fixed $\omega \in \Omega^{\prime}$ is a convex function that is identical with $\Phi(q)$ on $\mathscr{R}$, hence the two functions are identical on the completion of $\mathscr{R}$. Take a subsequence $\left\{m_{i}\right\}$ of $\mathscr{N}$ such that

$$
S_{m_{i}}\left(q_{0} \mid \omega\right) \rightarrow \liminf _{\mathscr{N} \ni n \rightarrow \infty} S_{n}\left(q_{0} \mid \omega\right):=a, \quad \omega \in \Omega^{\prime} .
$$

as $m_{i} \rightarrow \infty$. Let $r_{\alpha} \in \mathscr{R}, \alpha=1,2,3$ :

$$
r_{1}<q_{0}<r_{2}<r_{3}
$$

and

$$
P_{m \alpha}=\left(r_{\alpha}, S_{m}\left(r_{\alpha}\right)\right) \text {. }
$$

Since $S_{m}(q)$ is convex, the point $\left(q_{0}, S_{m}\left(q_{0}\right)\right)$ lies above the straight line that connects $P_{m 2}$ and $P_{m 3}$ and below the straight line that connects $P_{m 1}$ and $P_{m 2}$. However, $S_{m_{i}}\left(r_{\alpha}\right) \rightarrow \Phi\left(r_{\alpha}\right), i \rightarrow \infty$. For this reason the limiting point $\left(q_{0}, a\right)$ will have the same properties in relation to the lines that connect the corresponding pairs of points among the $\left(r_{\alpha}, \Phi\left(r_{\alpha}\right)\right)$.

The function $\Phi$ is smooth in $\left(q_{-}, q_{+}\right)$, while $\left\{r_{\alpha}\right\}$ can be chosen arbitrarily within $\mathscr{R}$. Since $\mathscr{R}$ is dense on $\left(q_{-}, q_{+}\right)$, then $a=\Phi\left(q_{0}\right)$.

Proposition 7. If $q_{\star}=q_{+}\left(q_{-}\right)$is a point of the second kind and $\left|q_{\star}\right|<\infty$, then under (3),

$$
\lim _{\mathscr{N} \ni n \rightarrow \infty} S_{n}(q)=q \cdot \Phi\left(q_{\star}\right) / q_{\star}, \quad \forall q: q / q_{\star}>1, \omega \in \Omega^{\prime} .
$$

If $q=0$, then the limit equals $\infty$ for $q<0$. Here $\mathscr{N}$ and $\Omega^{\prime}$ are the same as in Proposition 6.

Proof. Let $q_{\star}=q_{+}$for the sake of definiteness; $q_{0} \in\left(q_{-}, q_{+}\right)$and $q>q_{+}$. Similarly to the above (see (28)),

$$
\sum_{n}(q)=\sum_{1 \leqq i \leqq c^{n}}\left[\int_{\Delta_{i n}} P_{1}\left(t \mid w_{q_{0}}\right) \cdots P_{n}\left(t \mid w_{q_{0}}\right) P_{n}(t \mid \xi) d t\right]^{q / q_{0}} \cdot c_{n}
$$

where $\xi=M^{q_{0}} / E M^{q_{0}}$ and

$$
c_{n}=\left[c^{n \Phi\left(q_{0}\right)} E M^{q_{0}}\right]^{q / q_{0}} .
$$

Applying the inequality

$$
\sum_{i} x_{i}^{\lambda} \leqq\left(\sum_{i} x_{i}\right)^{\lambda}, \quad \lambda>1, x_{i} \geqq 0
$$

to (31) with $\lambda=q / q_{0}$, one gets

$$
\sum_{n}(q) \leqq c_{n} \cdot\left[M_{n}\left(w_{q_{0}}, \xi\right)\right]^{q / q_{0}} .
$$


It follows from the proof of Proposition 7 that

$$
\frac{1}{n} \log M_{n}\left(w_{q_{0}}, \xi\right) \rightarrow 0, \quad \mathscr{N} \ni n \rightarrow \infty
$$

for all $q_{0} \in\left(q_{-}, q_{+}\right)$and $\omega \in \Omega^{\prime}, P\left(\Omega^{\prime}\right)=1$.

Hence, for $\omega \in \Omega^{\prime}$,

$$
\limsup _{\mathscr{N} \ni n \rightarrow \infty} S_{n}(q) \leqq \lim _{n \rightarrow \infty} \frac{1}{n} \log c_{n}=\Phi\left(q_{0}\right) q / q_{0}
$$

The set $\Omega^{\prime}$ is independent of $q_{0}$, so one can pass to the limit as $q_{0} \rightarrow q_{+}$. Similarly we get (32) with $q_{0}=q_{-}<0$ for $q<q_{-}$.

The case $q_{-}=0$ will be discussed a little later.

Let us evaluate $S_{n}(q), n \in \mathscr{N}$ from below. Take a subsequence $\left\{m_{i}\right\} \subset \mathscr{N}$ such that

as $m_{i} \rightarrow \infty$.

$$
S_{m_{i}}(q) \rightarrow \liminf _{\mathscr{N} \ni n \rightarrow \infty} S_{n}(q)=a, \quad \omega \in \Omega^{\prime},
$$

Let $q_{\alpha} \in\left(q_{-}, q_{+}\right), \alpha=1,2$. Then $\left(q, S_{m}(q)\right)$ lies above the straight line that connects the points $\left(q_{\alpha}, S_{m}\left(q_{\alpha}\right)\right)$ for $q>q_{+}\left(q<q_{-}\right)$. Repeating the argument used to prove Proposition 6 , one obtains that $(q, a)$ will lie above the straight line that connects the points $\left(q_{\alpha}, \Phi\left(q_{\alpha}\right)\right), \alpha=1,2$ for $\mu(\cdot \mid \omega), \omega \in \Omega^{\prime}$. Hence

$$
\liminf _{\mathscr{N} \ni n \rightarrow \infty} S_{n}(q) \geqq \Phi\left(q_{1}\right)+\frac{\Phi\left(q_{2}\right)-\Phi\left(q_{1}\right)}{q_{2}-q_{1}}\left(q-q_{1}\right)
$$

for $\forall q \in(-\infty, q) \cup\left(q_{+}, \infty\right)$ and $\omega \in \Omega^{\prime}$.

Passing to the limit as $q_{2} \rightarrow q_{1}$ and then as $q_{1} \rightarrow q_{+}$, one gets

$$
\liminf _{\mathscr{N} \ni n \rightarrow \infty} S_{n}(q) \geqq \Phi\left(q_{+}\right)+\dot{\Phi}\left(q_{+}\right)\left(q-q_{+}\right) .
$$

Since $q_{+}$is a point of the second kind, it follows that $\dot{\Phi}\left(q_{+}\right) q_{+}=\Phi\left(q_{+}\right)$. Consequently, the upper (see (32)) and the lower bound of $S_{n}(q), n \rightarrow \infty$, are identical and equal to $\Phi\left(q_{+}\right) q / q_{+}$for all $q>q_{+}$and $\omega \in \Omega^{\prime}$.

For the case $q<q_{-}=0$ one has an uncommon situation. In that case $\dot{\Phi}\left(q_{0}\right) \rightarrow \infty$, $q_{0} \rightarrow q_{-}+0$. Consequently, one obtains from (33) that

$$
\limsup _{\mathscr{N} \ni n \rightarrow \infty} S_{n}(q) \geqq \liminf _{\mathscr{N} \ni n \rightarrow \infty} S_{n}(q)=\infty \text {. }
$$

Proposition 8. Under (3), there exist sets $\mathscr{N}, \Omega^{\prime}, \mathscr{P}\left(\Omega^{\prime}\right)=1$ such that (30) remains valid for points $q_{ \pm}$of the first kind.

Proof. Let $\mathcal{N}, \Omega^{\prime}$ be the same as in Proposition 6. The derivation of the upper bound (32) for $S_{n}(q), \mathscr{N} \ni n \rightarrow \infty, \omega \in \Omega^{\prime}$ did not use the type of $q_{ \pm}$. It follows that the bound still holds. We are going to evaluate $S_{n}(q)$ from below.

Let $q_{\star}=q_{+}\left(q_{-}\right)$be a point of the first kind, $q / q_{\star}>1$ and $q_{\star} \neq 0$. Put $q_{0}=q_{\star}$ in (31). All normalizing constants for points of the first kind are finite: $E w^{q \star}<\infty$, $E M^{q \star}<\infty$ (Proposition 5).

The functions $P_{i}\left(t \mid w_{q_{\star}}\right)$ in (31) are piecewise constant and $P_{i}\left(t \mid w_{q_{\star}}\right) \stackrel{d}{=} w_{q_{\star}}$ for a fixed $t$. The random variables $\zeta=w_{q_{\star}} / c$ have the following properties:

$$
c E \zeta=1 ; \quad E \zeta \log _{c} \zeta<0 ; \quad E \zeta^{\gamma}=\infty, \quad \forall \gamma>1 ; \quad P(\zeta>0)=1 .
$$


The first of these is obvious, the second is another form of (25), and the third means that $q_{\star}$ is a boundary of the domain of finite values of $\Phi(q)$. Points of the first kind, i.e. $q_{\star}$, have these properties.

From Lemma 9 (see below) it follows that under (34) there exists a transformation $\eta=f(\zeta)$ which depends on the parameter $\gamma \in\left(1, \gamma_{1}\right)$ such that

$$
\eta<\zeta^{\gamma}
$$

and

$$
c E \eta=1 ; \quad E \eta \log _{c} \eta<0 ; \quad E \eta^{h_{0}}<\infty, \quad h_{0}>1 ; \quad P(\eta>0)=1 .
$$

That means that $\sum_{n}(q)$ (see (28)) can be evaluated from below by making the substitution,

$$
c^{-\gamma} P_{m}^{\gamma}\left(t \mid w_{q_{\star}}\right) \rightarrow c^{-1} P_{m}(t \mid c \eta), \quad \gamma=q / q_{\star} \in\left(1, \gamma_{1}\right),
$$

that is, $P_{m}^{\gamma}(t)=a^{\gamma}$ is replaced with $f(a / c)$ in each interval $\Delta_{k m}, k=1, \ldots, c^{m}$, $m=1, \ldots, n$.

The sum $\sum_{n}(q)$ is decreased, when a similar operation is applied to $P_{n}^{\gamma}(t \mid \xi)$ :

$$
P_{n}^{\gamma}(t \mid \xi) \rightarrow P_{n}\left(t \mid \xi^{\prime}\right)
$$

where

$$
\xi^{\prime}= \begin{cases}\xi^{\gamma} & \text { if } \xi \leqq 1 \\ \xi & \text { if } \xi>1 .\end{cases}
$$

As a result, one gets for $q / q_{+} \in\left(1, \gamma_{1}\right)$,

$$
c_{n}^{-1} \sum_{n}(q)>\int P_{1}(t \mid c \eta) \cdots P_{n}(t \mid c \eta) P_{n}\left(t \mid \xi^{\prime}\right) d t=M_{n}\left(c \eta, \xi^{\prime \prime}\right) E \xi^{\prime}
$$

(see (31)) where $\xi^{\prime \prime}=\xi^{\prime} / E \xi^{\prime}, \xi^{\prime}>0$ a.s.,

$$
E \xi^{\prime}=E \xi^{\gamma}[\xi<1]+E \xi[\xi>1]<1+E \xi=2
$$

while $\eta$ satisfies (35).

By Proposition 2

$$
\underset{n \rightarrow \infty}{d-\lim _{n}} M_{n}\left(c \eta, \xi^{\prime \prime}\right)=Z,
$$

where $E Z=1, P(Z>0)=1$. Let $\mathscr{N}^{\prime} \subset \mathscr{N}$ and $\Omega^{\prime \prime} \subset \Omega^{\prime}$ be such that $\mathscr{P}\left(\Omega^{\prime \prime}\right)=1$ and $M_{n} / n \rightarrow 0$ as $\mathscr{N}^{\prime} \ni n \rightarrow \infty$ and $\omega \in \Omega^{\prime \prime}$.

Similarly to the above (see the proof of Proposition 7), we conclude that

$$
\liminf _{\mathcal{N}^{\prime} \ni n \rightarrow \infty} \frac{1}{n} \log \sum_{n}(q) \geqq \lim \frac{1}{n} \log c_{n}=\Phi\left(q_{+}\right) q / q_{+}
$$

for $q / q_{\star} \in\left(1, \gamma_{1}\right), \omega \in \Omega^{\prime \prime}$.

Recalling the upper bound (32), one gets

$$
\lim _{\mathscr{N}^{\prime} \in n \rightarrow \infty} S_{n}(q)=f\left(q_{\star}\right) q / q_{\star}
$$

for $q / q_{\star} \in\left(1, \gamma_{1}\right), \omega \in \Omega^{\prime \prime}, \mathscr{P}\left(\Omega^{\prime \prime}\right)=1$.

We are now in a situation that arose when proving Proposition 7 , the upper bound of $S_{n}(q)$ with $q / q_{+}>\gamma_{1}$ being determined by the curve $f\left(q_{\star}\right) q / q_{\star}$ and 
the lower by the tangent to the curve at $q=q_{\star}\left(\gamma_{1}-\varepsilon\right)$. Since these are identical, Proposition 8 is proven for $q_{\star} \neq 0$.

Case $q_{-}=0 . q_{-}$is a point of the first kind. Below we are using only the fact that $E w^{q}=\infty, \forall q<0$. When $q<0$,

$$
w^{q}>(w+a)^{q}, \quad a>0 ; \quad M^{q}>M^{\prime q}, \quad M^{\prime}=\max (M, \delta), \quad \delta>0 .
$$

Similarly to the above, we obtain from (28) for $q<0$,

$$
\sum_{n}(q)>\rho_{n} \int P_{1}^{q}\left(t \mid w^{\prime}\right) \cdots P_{n}^{q}\left(t \mid w^{\prime}\right) P_{n}^{q}\left(t \mid M^{\prime \prime}\right) \Delta_{n}^{q-1} d t
$$

where

$$
\begin{aligned}
w^{\prime} & =(w+a) /(1+a), \\
M^{\prime \prime} & =M^{\prime} / E M^{\prime}, \\
\rho_{n} & =(1+a)^{q n}\left(E M^{\prime}\right)^{q} .
\end{aligned}
$$

The right-hand side of (36) is, apart from the factor $\rho_{n}$, a sum of the type $\sum_{n}(q)$ constructed for the variables $w^{\prime}$ and $M^{\prime \prime}$, i.e.,

$$
\sum_{n}(q)>\rho_{n} \sum_{n}\left(q \mid w^{\prime}, M^{\prime \prime}\right) .
$$

Propositions 6 and 7 are applicable to the sums $\sum_{n}\left(q \mid w^{\prime}, M^{\prime \prime}\right)$. In fact, since $E w^{q}<\infty, \forall q<0$, it is only points $q_{-}(a)$ of the second kind which can occur on the negative $q$-axis.

The condition $E w^{\prime} \log _{c} w^{\prime}<1$ is satisfied for $0<a<a_{0}$, because

$E w^{\prime} \log w^{\prime}=(1+q)^{-1} E(w+a) \log (w+a)-\log (1+a) \rightarrow E w \log w<1, \quad a \rightarrow 0$.

Lastly, $E\left(M^{\prime \prime}\right)^{q}<\infty$, since $M^{\prime \prime}>\delta / E \max (M, \delta)$.

Thus, applying Propositions 6 and 7 to the right-hand side of (36), we get

$$
\liminf _{\mathcal{N}^{\prime \prime} \ni n \rightarrow \infty} S_{n}(q) \geqq q \cdot \log _{c}(1+a)+\Phi_{a}(q), \quad \omega \in \Omega^{\prime \prime}, q<0,
$$

where

$$
\Phi_{a}(q)= \begin{cases}\log _{c} E w^{\prime q}-q+1, & q>q_{-}(a) \\ \dot{\Phi}_{a}\left(q_{-}(a)\right) q, & q<q_{-}(a) .\end{cases}
$$

and $\mathscr{P}\left(\Omega^{\prime \prime}\right)=1$ and $\mathscr{N}^{\prime \prime} \subset \mathscr{N}$.

We now show that $\Phi_{a}(q) \rightarrow \infty$ as $a \rightarrow 0$. The straight line $y=\dot{\Phi}_{a}\left(q_{-}(a)\right) q$ supports $\Phi_{a}(q)$, and so

$$
\Phi_{a}(q)>\dot{\Phi}_{a}(q-(a)) q, \quad q<0 .
$$

Hence $\Phi_{a}(q) \rightarrow \infty$, if $\left|\dot{\Phi}_{a}\left(q_{-}(a)\right)\right| \rightarrow \infty, a \rightarrow \infty$.

Suppose $q_{-}(a) \rightarrow \infty, a \rightarrow 0$, then also $\Phi_{a}(q)=\log E w^{\prime q}-q+1 \rightarrow \infty$ (recall that $\left.E w^{q}=\infty\right)$. Suppose $\left|\dot{\Phi}_{a}\left(q_{-}(a)\right)\right|$ and $\left|q_{-}(a)\right|$ are bounded as $a \rightarrow 0$, while $\Phi_{a}(q) \nrightarrow \infty$. Choose a sequence $a_{n} \downarrow 0$ such that

$$
q_{-}\left(a_{n}\right) \rightarrow q_{-}^{0} \text {. }
$$


Obviously $q_{-}^{0}=0$. Otherwise $\Phi_{a_{n}}(q) \rightarrow \infty, a_{n} \rightarrow 0$ for small $|q|$ and is bounded for large $q$ (see (39)). This contradicts the convexity of $\Phi_{a}(q)$. Hence

$$
\Phi_{a}\left(q_{-}\left(a_{n}\right)\right)=\dot{\Phi}_{a}\left(q_{-}\left(a_{n}\right)\right) q_{-}\left(a_{n}\right) \rightarrow 0, \quad n \rightarrow \infty .
$$

However, $\Phi_{a}\left(q_{-}(a)\right)>\Phi_{a}(0)=1$. This is a contradiction.

Consequently, if $E w^{q}=\infty, \forall q<0$, then

$$
\liminf _{\mathcal{N}^{\prime \prime} \ni n \rightarrow \infty} S_{n}(q)=\infty
$$

since $a \in\left(0, a_{0}\right)$ is arbitrary.

Lemma 9. Let the random variable $\zeta$ satisfy

(i) $c E \zeta=1$;

(ii) $E \zeta \log _{c} \zeta=a<0$;

(iii) $E \zeta^{h}=\infty, \forall h>1$;

(iv) $P(\zeta>0)=1$.

Then there exists a transformation $\eta=f(\zeta)$ which depends on the parameter $\gamma$, such that $\eta$ has the properties (i,ii,iv), and also $E \eta^{1+\delta}<\infty, \delta>0$ and

$$
\eta<\zeta^{\gamma}, \quad 1<\gamma<\gamma_{1}
$$

Proof. We shall seek $\eta$ in the form

$$
\eta=d \cdot \begin{cases}\zeta^{1-\delta}, & \zeta>x_{0}>1 \\ \zeta_{\gamma}\left(x_{0}\right), & \zeta<x_{0}\end{cases}
$$

where $\zeta_{\gamma}\left(x_{0}\right)=\zeta^{\gamma} / m_{\gamma}$,

$$
m_{\gamma}=E \zeta^{\gamma}\left[\zeta<x_{0}\right] \text {. }
$$

We choose $x_{0}>1$, so that $m_{\gamma}>1$. This is possible, since $m_{\gamma} \rightarrow \infty, x_{0} \rightarrow \infty$ (property (iii)).

Then, for $d<1$,

$$
\eta=d / m_{\gamma} \cdot \zeta^{\gamma}<\zeta^{\gamma}, \quad \zeta<x_{0} .
$$

On the other hand, when $0<\delta<1, \gamma>1$,

$$
\eta=d \zeta^{1-\delta}<\zeta^{1-\delta}<\zeta^{\gamma}, \quad \zeta>x_{0}>1 .
$$

It follows that $\eta<\zeta^{\gamma}$ and $E \eta^{1 /(1-\delta)}<\infty$.

Condition (i) for $\eta$ defines $d$ :

$$
d=c^{-1}\left(1+E \zeta^{1-\delta}\left[\zeta>x_{0}\right]\right)^{-1}<1 .
$$

Condition (ii) for $\eta$ reduces to

$$
A(\gamma)=E \zeta_{\gamma}\left(x_{0}\right) \log _{c}\left(d \zeta_{\gamma}\left(x_{0}\right)\right) \cdot\left[\zeta<x_{0}\right]+E \zeta^{1-\delta} \log _{c} d \zeta^{1-\delta}\left[\zeta>x_{0}\right]<0 .
$$

We now show that the requirement $A(\gamma)<0$ can be satisfied by a suitable choice of $x_{0}>1$ and $\gamma>1$.

The first term in $A(\gamma)$ is

$$
J(\gamma):=E \zeta_{\gamma}\left(x_{0}\right) \log _{c} \zeta_{\gamma}\left(x_{0}\right)\left[\zeta<x_{0}\right]+\log _{c} d .
$$


This function increases with increasing $\gamma$. In fact

$$
J(\gamma)=\gamma \dot{r}(\gamma)-r(\gamma)+\log _{c} d,
$$

where

$$
r(\gamma)=\log _{c} E \zeta^{\gamma}\left[\zeta<x_{0}\right]
$$

and

$$
\dot{J}(\gamma)=\gamma \ddot{r}(\gamma) \geqq 0, \quad \gamma>0
$$

Since

$$
J(1)=m_{1}^{-1} E \zeta \log \zeta\left[\zeta<x_{0}\right]+\log \left(d / m_{1}\right),
$$

and

$$
\begin{aligned}
m_{1}=E \zeta\left[\zeta<x_{0}\right] & \rightarrow E \zeta=c^{-1}, \quad x_{0} \rightarrow \infty, \\
d & \rightarrow c^{-1}, \quad x_{0} \rightarrow \infty,
\end{aligned}
$$

then $J(1) \rightarrow c E \zeta \log _{c} \zeta=c a<0, x_{0} \rightarrow \infty$. The second term in $A(\gamma)$ vanishes as $x_{0} \rightarrow \infty$. Therefore

$$
A(1)<-b<0, \quad \forall x_{0}>x_{0}^{\star} .
$$

Take some $x_{0}>x_{0}^{\star}, x_{0}>1$. $A(\gamma)$ is a smooth function of $\gamma$ in a vicinity of $\gamma=1$ and is increasing. Hence $A(\gamma)<0$ in some interval $1<\gamma<\gamma_{0}$.

\section{Multifractal Dimensions for $\mathscr{M}$-Cascades: Proof of Theorem 2}

The proof uses the following.

Proposition 10 [9]. Let $M$ be the total mass of $\mathscr{M}$-cascade measure $\mu$. If $E M \log _{c} M<\infty$, then with probability 1 ,

$$
\lim _{n \rightarrow \infty}-\frac{1}{n} \log _{c} \mu\left(\Delta_{n}(t)\right)=D=1-E w \log _{c} w \quad \mu \text {-a.s. },
$$

where $\Delta_{n}(t)$ is the sequence of intervals $\Delta_{\text {in }}$ that contain $t$.

Proof of Theorem 2. Lower bound. Let $q_{-} \neq 0$ and

$$
\alpha=-\dot{\Phi}(q), \quad q \in\left(q_{-}, q_{+}\right), q \neq 0 .
$$

The value $q=0$ is ruled out, because $f(\alpha) \neq 1$.

By (28)

$$
\mu^{q}\left(\Delta_{n}(t)\right)=P_{1}\left(t \mid w_{q}\right) \cdots P_{n}\left(t \mid w_{q}\right) \Delta_{n} P_{n}\left(t \mid M^{q}\right) \cdot c_{n},
$$

where $w_{q}=w^{q} / E w^{q}$ and

$$
c_{n}=c^{\Phi(q) n} .
$$

According to Proposition 5, there exists, for $w_{q}$, a cascade measure $\mu_{q}(d t)$ with total mass $M_{q}$ such that

$$
M_{q}>0 \text { a.s., } \quad E M_{q}^{h}<\infty, \forall h \in\left(1, h_{0}\right) .
$$

Therefore (41) can be written as

$$
\mu^{q}\left(\Delta_{n}(t)\right)=\mu_{q}\left(\Delta_{n}(t)\right) P_{n}\left(t \mid M^{q}\right) / P_{n}\left(t \mid M_{q}\right) \cdot c_{n},
$$


where $P_{n}\left(t \mid M_{q}\right)$ and $P_{n}\left(t \mid M^{q}\right)$ are measurable with respect to the $\sigma$-algebra $\sigma\left\{P_{n}(t), m>n\right\}$ and hence are independent of $P_{i}(t), i \leqq n$.

Since $E M_{q}^{h}<\infty, h \in\left(1, h_{0}\right)$, this implies that

$$
E M_{q}\left|\log M_{q}\right|^{\rho}<\infty, \quad \forall \rho>0 .
$$

Consequently, applying Proposition 10 to $\mu_{q}$, one gets

$$
\lim _{n \rightarrow \infty}-\frac{1}{n} \log _{c} \mu_{q}\left(\Delta_{n}(t)\right)=D(q)=\Phi(q)-\dot{\Phi}(q) q, \quad \mu_{q} \text {-a.s } .
$$

We shall show that, with probability 1 ,

as $n \rightarrow \infty$.

$$
f_{n}(t):=\frac{1}{n} \log P_{n}\left(t \mid M_{q}\right) \rightarrow 0, \quad \mu_{q} \text {-a.s } .
$$

Let

$$
Q(d t, d \omega)=\mu_{q}(d t) \mathscr{P}(d \omega)
$$

be the Peyrière probability [9]. By Chebyshev's inequality

$$
Q\left(\left|f_{n}(t \mid \omega)\right|>\varepsilon\right)<(\varepsilon \cdot n)^{-2} E \eta_{n},
$$

where

$$
\begin{aligned}
\eta_{n} & =n^{2} \int f_{n}^{2}(t) \mu_{q}(d t) \\
& =\int P_{1}\left(t \mid w_{q}\right) \cdots P_{n}\left(t \mid w_{q}\right) P_{n}\left(t \mid M_{q}\right)\left|\log P_{n}\left(t \mid M_{q}\right)\right|^{2} d t \\
& =\int P_{1}\left(t \mid w_{q}\right) \cdots P_{n}\left(t \mid w_{q}\right) P_{n}(t \mid \xi) d t
\end{aligned}
$$

and

$$
\xi=M_{q}\left|\log M_{q}\right|^{2} \text {. }
$$

Since $P_{1}\left(t \mid w_{q}\right), \ldots, P_{n}\left(t \mid w_{q}\right), P_{n}(t \mid \xi)$ are independent,

$$
E \eta_{n}=E \xi<\infty \text {. }
$$

Using the convergence of

$$
\sum_{n \geqq 1} E \eta_{n} / n^{2}=E \xi \sum_{n \geqq 1} n^{-2}<\infty
$$

and the Borel-Cantelli lemma, one concludes that

$$
f_{n}(t \mid \omega) \rightarrow 0, \quad Q \text {-a.s. }
$$

as $n \rightarrow \infty$, whence (44).

Similarly one can show that with probability one

$$
\varphi_{n}(t):=\frac{1}{n} \log P_{n}\left(t \mid M^{q}\right) \rightarrow 0, \quad \mu_{q} \text {-a.s } .
$$

as $n \rightarrow \infty$. In that case

$$
\xi=q^{2} M_{q}|\log M|^{2} .
$$

To show that $E \xi<\infty$, we use the Hölder inequality

$$
E \xi<q^{2}\left(E M_{q}^{h}\right)^{1 / h}\left(E|\log M|^{2 h^{\prime}}\right)^{1 / h^{\prime}}, h^{-1}+\left(h^{\prime}\right)^{-1}=1,
$$


where $h \in\left(1, h_{0}\right)$. The right-hand side of (46) is finite for any $h^{\prime}$, if $E M^{-\varepsilon}<\infty$ for some $\varepsilon>0$. The last requirement is obviously true, if $q_{-}<0$ (Proposition 4).

Using (42) together with (43-45), one obtains, with probability one,

$$
\begin{aligned}
q \cdot \lim _{n \rightarrow \infty}-\frac{1}{n} \log \mu\left(\Delta_{n}(t)\right) & =\lim _{n \rightarrow \infty}\left[-\frac{1}{n} \log \mu_{q}\left(\Delta_{n}(t)\right)-\varphi_{n}(t)+f_{n}(t)\right]-\Phi(q) \\
& =[\Phi(q)-\dot{\Phi}(q)]-\Phi(q)=\alpha q \quad \mu_{q} \text {-a.s } .
\end{aligned}
$$

However, $\mu_{q}$ has a carrier of dimension $D(q)=\Phi(q)-\dot{\Phi}(q) q=f(\alpha)$, and so

$$
H-\operatorname{dim} J_{\alpha} \geqq H-\operatorname{dim}\left(\operatorname{supp} \mu_{q}\right)=f(\alpha) \text {. }
$$

The case $q_{-}=0$. Here, it is only the conclusion about the convergence $\varphi_{n}(t) \rightarrow 0$ which needs modification. Let $E|\log w|^{\delta}<\infty$. Then, by Proposition 4, $E|\log M|^{\delta^{\prime}}<\infty, \delta^{\prime}>0$. Therefore, if

$$
\xi_{\rho}=M_{q}|\log M|^{\rho},
$$

then

$$
E \int \varphi_{n}^{\rho}(t) \mu_{q}(d t)=n^{-\rho} q^{\rho} E \xi_{\rho}
$$

and

$$
E \xi_{\rho}<\left(E M_{q}^{h}\right)^{1 / h}\left(E|\log M|^{h^{\prime} \rho}\right)^{1 / h^{\prime}}<\infty
$$

if $h^{\prime} \rho<\delta^{\prime}$. Therefore $\varphi_{n}(t \mid \omega) \rightarrow 0 Q$-a.s. as $n \rightarrow \infty$ and $n \in \mathscr{N}=\left\{n_{i}: n_{i+1} / n_{i} \geqq\right.$ $k>1$ \}. Using the above arguments we get

$$
H-\operatorname{dim} J_{\alpha}(\mathscr{N}) \geqq f(\alpha), \quad \text { a.s } .
$$

Proof of Theorem 2. Upper bound. The proof is general and relies on the existence of the $\tau$-function, which is ensured by Theorem 1 . The proof has some intersections with that of Lemma 3.4 [2].

Let $\Omega^{\prime}$ and $\mathcal{N}$ be sets of events and integer numbers for which Theorem 1 holds and $M=M(\omega)>0$. Then for fixed $\omega \in \Omega^{\prime}$,

$$
\sum_{i} \mu^{q}\left(\Delta_{i n}\right)=\Delta_{n}^{\tau(q)} \varphi_{n}(q)
$$

where

$$
n^{-1} \log \varphi_{n} \rightarrow 0, \quad \mathscr{N} \ni n \rightarrow \infty \forall q:|\tau(q)|<\infty
$$

Let

$$
G_{n}(\beta)=\left\{\Delta_{\text {in }}: \mu\left(\Delta_{\text {in }}\right)>\Delta_{n}^{\beta}\right\}
$$

and $N_{n}(\beta)$ be the number of intervals $\Delta_{\text {in }}$ in $G_{n}(\beta)$.

By Chebyshev's inequality

$$
N_{n}(\beta)<\sum_{i} \mu^{q}\left(\Delta_{i n}\right) / \Delta_{n}^{q \beta}=\Delta_{n}^{\tau(q)-q \beta} \varphi_{n} .
$$

Let $q$ be such that

$$
q \beta-\tau(q)=\min _{h}(h \beta-\tau(h))=f(\beta) .
$$

Then

$$
N_{n}(\beta)<\Delta_{n}^{-f(\beta)} \varphi_{n}<\Delta_{n}^{-(f(\beta)+\delta)}, \quad \forall \delta>0, \mathscr{N} \ni n>N(\delta) .
$$

If $\beta>\alpha$, then

$$
J_{\alpha}(\mathscr{N}) \subset \bigcap_{n \in \mathcal{N}} \bigcup_{\mathscr{N} \ni m \geqq n} G_{m}(\beta)=G_{\beta}
$$


and

$$
H-\operatorname{dim} J_{\alpha} \leqq H-\operatorname{dim} G_{\beta}
$$

We now show that

$$
H-\operatorname{dim} G_{\beta} \leqq f(\beta)
$$

which implies (13), since $\beta>\alpha$ is arbitrary.

By definition, the $f$-Hausdorff outer measure of set $G$ is

$$
H_{f}(G)=\lim _{\varepsilon \rightarrow 0} H_{f}^{\varepsilon}(G)
$$

where

$$
H_{f}^{\varepsilon}(G)=\inf \sum\left|\Delta_{i}\right|^{f}
$$

and the infimum is taken over all $\varepsilon$-coverings of $G$. $H_{f}^{\varepsilon}$ increases as $\varepsilon \rightarrow 0$, hence, for $\varepsilon: \varepsilon_{n}=c^{-n}$,

$$
H_{f}^{\varepsilon_{n}}\left(G_{\beta}\right)<H_{f}^{\varepsilon_{n}}\left(\bigcup_{\mathcal{N} \ni m \geqq n} G_{m}(\beta)\right)<\sum_{\mathcal{N} \ni m \geqq n} H_{f}^{\varepsilon_{m}}\left(G_{m}(\beta)\right) .
$$

By virtue of (47)

$$
H_{f}^{\varepsilon_{n}}\left(G_{n}(\beta)\right)<2 N_{n}(\beta) \Delta_{n}^{f}<2 \Delta_{n}^{(f-f(\beta)-\delta)}, \quad n \in \mathscr{N} .
$$

Putting $f=f(\rho)+\delta^{\prime}, \delta^{\prime}>\delta$, we conclude that the right-hand side of (49) vanishes as $n \rightarrow \infty$. Therefore $H_{f}\left(G_{\beta}\right)=0$ and (see [4]) $H-\operatorname{dim} G_{\beta} \leqq f=f(\rho)+\delta^{\prime}$. Since $\delta^{\prime}>0$ is arbitrary, we get (48).

\section{Cascades of the General Type: Proof of Theorems 3, 4}

Theorem 1 relies on a sequence of propositions that need some explanation in this new situation.

Proposition 1. In the equation for mass $M$,

$$
M=\sum M_{i} w_{i} / c
$$

$\left\{M_{i}\right\}$ are independent copies of $M$ that are independent of $W=\left(w_{1}, \ldots, w_{c}\right)$.

Propositions 2, 3 constitute Theorems 2(b) and 3 in [3].

Proposition 4 is modified as follows:

Under (2) the following implications are true for $W$ :

(a) $E w^{-h} \Rightarrow E M^{-h}<\infty, \quad h>0$,

(b) $E|\log w|^{h}<\infty \Rightarrow E|\log M|^{h^{\prime}}<\infty, \quad \forall h^{\prime}<h, h>0$,

where $w$ is the random component of $W$.

In fact, one gets using (50),

$$
M^{-p} \leqq \prod_{1 \leqq i \leqq c} M_{i}^{-p / c} \prod_{1 \leqq i \leqq c} w_{i}^{-p / c} \leqq \prod M_{i}^{-p / c} \sum_{i} w_{i}^{-p} / c, \quad p>0
$$

and so

$$
E M^{-p}<\left(E M^{-p / c}\right)^{c} E w^{p}
$$


It remains to establish the finiteness of $E M^{-\varepsilon}$ for some $\varepsilon>0$. Let

$$
\varphi(s)=E \exp (-s M) .
$$

By (50)

$$
\varphi(s)=E \prod_{1 \leqq i \leqq c} \varphi\left(s w_{i} / c\right) \leqq E \frac{1}{c} \sum_{i} \varphi^{c}\left(s w_{i} / c\right)=E \varphi^{c}(s w / c) .
$$

Further, similarly to the above,

$$
\varphi\left(s^{2}\right)<P\left(w / c<s^{-1}\right)+\varphi^{c}(s) .
$$

Under (a),

$$
\varphi\left(s^{2}\right)<p\left[s^{-h^{\prime}}+\varphi^{2}(s)\right], \quad s>s_{0}, h^{\prime}<h, \quad c>2,
$$

while under (b),

$$
\varphi\left(s^{2}\right)<p\left[(\ln s)^{-h^{\prime}}+\varphi^{c^{\prime}}(s)\right], \quad h^{\prime}<h, c^{\prime}>c .
$$

We have arrived at the inequalities considered above (see (19)).

Propositions 5-8 use Theorem 4 in the weak form only, i.e. with the parameter $a=1$. The formulations and proofs of Propositions 6-9 are preserved, if $w_{q}$ is understood as the vector

$$
W_{q}=\left(w_{1}^{q}, \ldots, w_{c}^{q}\right) / E w^{q},
$$

and as the random component of $W_{q}$ when integrating on $\mathscr{P}(d \omega)$. Rearranging the $W_{q}$ in Proposition 8 , we replace $W_{q}$ with $\zeta=\left\{\zeta_{i}\right\}$, while when dealing with mean values ((34), say), we also imply the random component of $\zeta$.

Theorem 2(a) is based on Proposition 10 which is applicable to $(\mu, W)$ unchanged (see, [11]).

Theorem 2(b). The proof totally depends on Theorem 1.

Acknowledgements. I thank D. Schertzer for stimulating discussions and U. Frisch for the hospitality at the Observatoire de la Cote d'Azur.

The work was supported by the French Ministry of Higher Education and in part by the Russian Foundation of Fundamental Research (Grant 96-01-00377) and by NSF (Grant EAR-9423818).

\section{References}

1. Collet, P., Koukiou, F.: Large deviations for multiplicative chaos. Commun. Math. Phys. 147, 329-342 (1992)

2. Collet, P., Lebowitz, J., Porzio, A.: The dimension spectrum of some dynamical systems. J. Stat. Phys. 47(5/6), 609-644 (1987)

3. Durrett, R., Liggett, T.M.: Fixed points of the smoothing transformation. Z. Wahr. Verw. Geb. 64, 275-301 (1983)

4. Falconer, K.J.: The geometry of fractal sets. Cambridge: Cambridge Univ. Press, 1985

5. Feller, W.: An introduction to probability theory and its applications. Vol. 2, New York: Wiley, 1966

6. Guivarc'h, Y.: Sur une extension de la notion de loi semistable. Ann. Inst. H. Poincarè 26(2), 261-285 (1990)

7. Holley, R., Liggett, T.: Generalized potlatch and smoothing processes. Z. Wahr. Verw. Geb. 55, 165-195 (1981) 
8. Holley, R., Waymire, E.: Multifractal dimensions and scaling exponents for strongly bounded random cascades. Ann. Appl. Probab. 2(4), 819-845 (1992)

9. Kahane, J.P., Peyrière, J.: Sur certaines martingale de B. Mandelbrot. Adv. Math. 22, 131-145 (1976)

10. Mandelbrot, B.: Multiplications alèatoires et distributions invariantes par moyenne pondèrèe alèatorie. C.R. Acad. Sci., Paris Ser. A, 278, 289-292, 355-358 (1974)

11. Nasr, Ben: Mesures aléatoires de Mandelbrot associées à des substitutions. C.R. Acad. Sci. Paris Ser. I, 304, 255-258 (1987)

12. Novikov, E.: Infinitely divisible distributions in turbulence. Phys. Rev. E 50(5), R3303-3305 (1994)

13. Peckman, S., Waymire, E.: On a symmetry of turbulence. Commun. Math. Phys. 147, 365-370 (1992)

14. Schertzer, D., Lovejoy, S.: Lecture Notes. AGU Chapman/ EGS Richardson Memorial Conference. Nonlinear variability in Geophysics 3. Scaling and multifractal processes. Inst. d'études sci. de Cargése (1993)

15. Sreenivasan, K., Stolovitzky, G.: Turbulent cascades. J. Stat. Phys. 78(1/2), 311-333 (1995)

16. She, Z.-S., Leveque, E.: Universal scaling laws in fully developed turbulence. Phys. Rev. Lett. 72(3), 336-339 (1994)

17. Waymire, E., Williams, S.: A cascade decomposition theory with applications to Markov and exchangeable cascades. Trans. Amer. Math. Soc. 348(2), 586-632 (1996)

Communicated by Ya.G. Sinai 